\title{
Vorteil der irreversiblen EGFR Hemmung
}

Für jene Formen des nicht-kleinzelligen Lungenkrebs (NSCLC), die Mutationen des aktivierten Epidermalen Wachstumsfaktorrezeptors = EGFR aufweisen, stehen seit Kurzem die EGFR Tyrosinkinasehemmer (TKI) als therapeutische Möglichkeit zur Verfügung. Der Zweitgenerations-TKI Afatinib hemmt eine größere Bandbreite an Prozessen in der Signalkaskade als die Erstgenerations-TKI und erreicht damit eine höhere Wirksamkeit, wie nun die Ergebnisse der LUX-Lung 7-Studie zeigen.

Erlotinib, Gefitinib und Afatinib sind als Erstlinientherapie für Patienten mit EGFR-Mutations-positivem NSCLC zugelassen. Gefitinib und Erlotinib zählen zur ersten Generation der TKIs und hemmen EGFR reversibel, während Afatinib als Blocker der ErbB-Familie eine irreversible EGFR-Hemmung bewirkt. In den beiden großen Phase III-Studien LUX-Lung 3 und 6 erwies sich Afatinib hinsichtlich seiner Wirksamkeit für ein progressionsfreies Überleben durchgehend als überlegen gegenüber der Standard Therapie mit Platin-basierter Chemotherapie [1, 2]. Auch bei Patienten mit Exon 19 Deletionsmutationen war das Gesamtüberleben (OS) signifikant erhöht, allerdings nicht bei L858R Mutationen [3].

\section{Direkter Vergleich in LUX-Lung 7}

Direkte Vergleiche zwischen den Erstlinien TKIs fehlen bisher. Mit der globalen, randomisierten Phase III Studie LUX-Lung 7 liegt nun eine solche direkte Vergleichsstudie für Afatinib gegenüber Gefitinib bei unbehandelten Patienten mit IIIB/IV Adenokarzinomen der Lunge und EGFR Mutation Exon 19- und L858R-Deletion - vor [4]. LUX-Lung 7 wurde in 64 Zentren in 13 Ländern in Nordamerika, Europa, Asien und Australien durchgeführt. Die Teilnehmer erhielten entweder Afatinib 40 mg einmal täglich oder Gefitinib 250 mg einmal täglich. Die drei primären Endpunkte waren: PFS, die Zeit bis zum Therapieversagen (TTF) und das Gesamtüberleben (OS). Die erste Analyse wurde beim ESMO Asia 2015 präsentiert.

Beim primären Endpunkt PFS war Afatinib mit 11,0 gegenüber 10,9 Mo- naten und einem Hazard ratio von $0,73(p=0,0165)$ signifikant überlegen. Dieser Vorteil war allerdings nicht gleich zu Beginn ersichtlich, sondern die PFS-Kurven entfernten sich erst im Verlauf der Behandlung voneinander. Nach zwölf und nach 24 Monaten zeigten sich deutliche Unterschiede zwischen den beiden Gruppen. Der Vorteil im PFS mit Afatinib wurde über alle Subgruppen beobachtet und war nicht durch den Mutationstyp oder andere Faktoren beeinflusst.

Beim zweiten ko-primären Endpunkt TTF zeigte die Analyse, dass ein höherer Anteil der Patienten in der Afatinib-Grupp die Behandlung fortsetzten (13,7 vs. 11,5 Monate; HR 0,73, $\mathrm{p}=0,0073)$. Dieser Unterschied bestätigt den Zuwachs an PFS und legt einen höheren Vorteil von Afatinib hinsichtlich des Anteils der Patienten nahe, die die Therapie beibehalten. Die Daten zum OS waren in dieser Analyse noch nicht zur Auswertung bereit, werden aber im Laufe dieses Jahres erwartet.

\section{Weitere Endpunkte}

Die objektive Ansprechrate lag in der Afatinib Gruppe bei $70 \%$ verglichen mit $56 \%$ in der Gefitinibgruppe $(p=0,0083)$. Auch die durchschnittliche Ansprechdauer war in der Afatinibgruppe länger als im Gefitinib-Arm mit 10,1 versus 8,4 Monaten. Bei Patienten mit einer Exon 19 Mutationsdeletion führte Afatinib zu deutlichen Vorteilen beim PFS (HR 0,76), bei der Ansprechrate (73 vs $66 \%$ ) und bei der Tumorreduktion. Diese Auswirkungen wurden in der Subgruppe

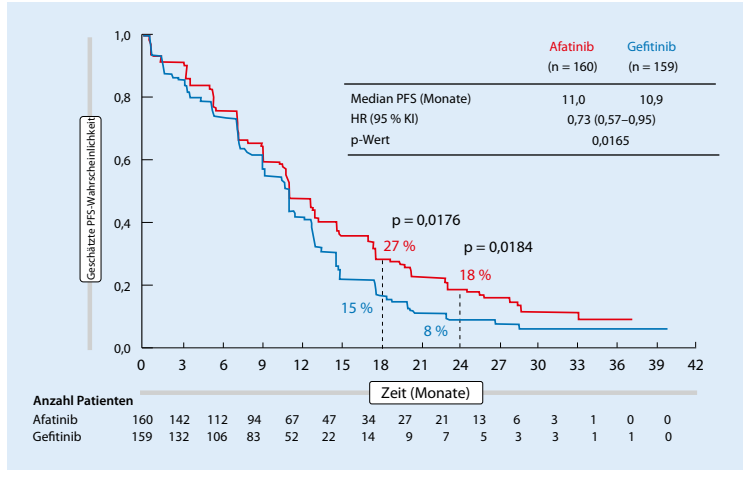

A PFS Vorteil von Afatinib verglichen mit Gefitinib in LUX-Lung 7 [4]

mit L858R-Mutatuioenen sogar noch übertroffen.

Die Ergebnisse zeigen auch die gute Kontrollierbarkeit der Nebenwirkungen dieser Substanzklasse.

\section{Fazit}

Die LUX-Lung 7 Studie bestätigt die Vorteile der irreversiblen ErbB-Blockade mit Afatinib verglichen mit der reversiblen EGFR-Hemmung mit Gefitinib in der Behandlung von EGFR-Mutations-positivem NSCLC.

\footnotetext{
Literatur

1. Sequist LV et al (2013) J Clin Oncol 31:3327-3334

2. Wu YL et al (2014) Lancet Oncol 15:213-222

3. Yang JC et al (2015) Lancet Oncol 16:141151

4. Park K et al (2015) (abstract LBA2_PR)
}

Wien klin Mag 2016 · 19:29

DOI 10.1007/s00740-016-0098-1

Online publiziert: 2. Februar 2016

C) Springer-Verlag Wien 2016 